\title{
Application of Parallel Liquid \\ Chromatography/Mass Spectrometry for High Throughput Microsomal Stability Screening of Compound Libraries
}

\author{
Rongda Xu, Csaba Nemes, Kelly M. Jenkins, Robyn A. Rourick, \\ and Daniel B. Kassel \\ DuPont Pharmaceuticals Research Laboratories, San Diego, California, USA
}

Charles Z. C. Liu

Applied Biosystems, Foster City, California, USA

\begin{abstract}
Solution-phase and solid-phase parallel synthesis and high throughput screening have enabled biologically active and selective compounds to be identified at an unprecedented rate. The challenge has been to convert these hits into viable development candidates. To accelerate the conversion of these hits into lead development candidates, early assessment of the physicochemical and pharmacological properties of these compounds is being made. In particular, in vitro absorption, distribution, metabolism, and elimination (ADME) assays are being conducted at earlier and earlier stages of discovery with the goal of reducing the attrition rate of these potential drug candidates as they progress through development. In this report, we present an eight-channel parallel liquid chromatography/mass spectrometry (LC/MS) system in combination with custom Visual Basic and Applescript automated data processing applications for high throughput early ADME. The parallel LC/MS system was configured with one set of gradient LC pumps and an eight-channel multiple probe autosampler. The flow was split equivalently into eight streams before the multiple probe autosampler and recombined after the eight columns and just prior to the mass spectrometer ion source. The system was tested for column-to-column variation and for reproducibility over a $17 \mathrm{~h}$ period (approximately 500 injections per column). The variations in retention time and peak area were determined to be less than 2 and 10\%, respectively, in both tests. The parallel LC/MS system described permits time-course microsomal incubations $\left(t_{0}, t_{5}, t_{15}, t_{30}\right)$ to be measured in triplicate and enables estimations of $t_{1 / 2}$ microsomal stability. The parallel LC/MS system is capable of analyzing up to 240 samples per hour and permits the complete profiling up to two microtiter plates of compounds per day (i.e., 176 test substrate compounds + sixteen controls). (J Am Soc Mass Spectrom 2002, 13, 155-165) @ 2002 American Society for Mass Spectrometry
\end{abstract}

A dvances in directed parallel synthesis and high throughput screening (HTS) have enabled large numbers of biochemically potent (active) and selective compounds to be identified at early stages of drug discovery [1-3]. However, the fact that a compound is active and selective does not necessarily make it an attractive drug development candidate. To convert these lead candidates into druggable molecules has proved elusive. It has been reported that a disproportionately large number of compounds entering devel-

Published online December 3, 2001

Address reprint requests to Dr. D. B. Kassel, DuPont Pharmaceuticals Research Labs, 4570 Executive Drive, Suite 400, San Diego, CA 92121, USA. E-mail: daniel.b.kassel@dupontpharma.com

*Present address: 3M Pharmaceutical ARD, St. Paul, MN 55144, USA. opment fail because of poor pharmacokinetics (nearly $40 \%$ ) [3-5]. Consequently, it has been recognized that pharmacokinetic studies that assess absorption, distribution, metabolism, and elimination (ADME) should be initiated as early as possible in the discovery process in order to maximize the likelihood of development success and minimize development costs. More and more, discovery programs are taking advantage of high throughput in vitro assays and property-based design tools to assist medicinal chemists in making not only potent and selective compounds, but ADME-friendly compounds as well [5].

The properties of absorption and metabolism have received perhaps the greatest amount of attention at early stages of discovery for the reason that oral dosing is by far the preferred route of administration to treat 
chronic illnesses and diseases. As compounds are administered orally, they are transferred across the intestinal lumen (the process of oral absorption) into the portal vein. Subsequently, these compounds are exposed to the liver (the major organ of xenobiotic transformation) prior to entering systemic circulation. It is in the liver where the drug is subject to first pass effects. The compound may be either a substrate for or an inhibitor of the cytochrome $\mathrm{P}_{450}$ metabolizing enzymes (i.e., the monooxidases primarily responsible for metabolizing xenobiotics). The extent to which a compound is a substrate for these enzymes influences in part the duration of action of the compound. Knowledge of the inhibitory effect of a drug molecule on any one of the key cytochrome $\mathrm{P}_{450}$ metabolizing enzymes is crucial to prevent undesirable drug-drug interactions when drugs are coadministered. Another very important reason absorption and metabolism are acknowledged as key properties to profile for at earlier stages of discovery is that they are the most well established (experimentally) and most well validated in vitro assays. In particular, the in vitro microsomal stability assay has been routinely used for the prediction of intrinsic clearance [6], a key pharmacokinetic property influencing in vivo half-life.

Liquid chromatography-mass spectrometry (LC/ MS) has become the preferred tool for in vitro ADME screens because of its sensitivity, selectivity, versatility, and ease of automation. Korfmacher et al. recently described a fully automated system for quantitative analysis of liver microsomal incubation samples incorporating LC/MS and automated data processing tools [7]. Although their method was automated, sample throughput was limited because of the relatively long HPLC gradient analysis times (10 min per sample). More recently, analysis throughput has been improved by shortening the HPLC run time [8-11]. Samples can be analyzed one at a time in as little as about one min per sample by applying fast gradients with most stateof-the-art LC/MS based techniques used to assess ADME properties [8]. The cycle time may be reduced further to less than one min when multiple autosamplers are used in concert to eliminate delays associated with sample loading and when preanalysis extraction and isocratic elution techniques are employed [10, 11]. However, in each of these single column systems, the samples are processed in serial, ultimately limiting the throughput and leaving little room for stretching the hardware capabilities if higher throughput is required.

Pooling strategies have been incorporated as a means for increasing throughput and reducing the total number of samples to be analyzed. Stevenson et al. demonstrated the power of this approach for the in vitro cell permeability screening of compound libraries [12]. Another approach is to dose multiple compounds together, known as cassette dosing. Berman and coworkers described how cassette dosing is applied to rapid pharmacokinetic screening [13]. Pooling strategies and cassette dosing are compromised principally by sensitivity and solubility issues (the larger the number of compounds pooled, the lower the concentration of each individual component possible) and the greater the potential for synergistic and/or antagonistic effects. Alternatively, multiple pharmacokinetic samples of an individual compound across all time points may be pooled before analysis [14].

A staggered injection and elution technique that uses multiple columns in parallel and acquires data only in the useful part of chromatograms can reduce overall analysis time as well [15-17]. Houda and co-workers recently demonstrated how fast LC/MS can be used to increase metabolic screening analysis throughput [18] by this method. This approach requires complex tubing and valve switching schemes. The staggered injection and elution method, although well suited to pharmacokinetic studies, has not yet proven useful for combinatorial library screening, where each member of the library has a unique structure and a unique chromatographic retention time.

True parallel approaches, on the other hand, are showing promise for high throughput ADME screening. The parallel LC/MS methods allow multiple samples to be analyzed in parallel by injecting discrete compounds onto multiple columns and detecting them simultaneously in a single mass spectrometer ion source $[6,19-26]$. One example is the recently commercialized multiplexed (MUX) electrospray interface, which introduces multiple LC flows directly into a multiplexed electrospray ion source [25, 27]. The technique has been shown to be extremely powerful for increasing qualitative analysis throughput. A challenge remains in developing automated data processing tools to facilitate postdata acquisition processing of MUXderived ADME data. An important limitation of the MUX technique at this time is that it is not ideally suited to quantitative analysis because of the sampling efficiency of the multiplexed sprayer assembly. As the number of sprayers increases, the duty cycle increases, leading to less frequent sampling at any given channel and hence, poorer ion statistics and greater error in mass measurements.

Our group has put great effort into developing parallel LC/MS technology in areas of compound library analysis, high throughput mass-directed purification, and ADME studies [19, 27-29]. In this report, an eight-channel parallel LC/MS system and associated VisualBasic and Applescript automated data processing applications for in vitro microsomal stability screening are discussed. The system was designed with easy implementation in mind so that an existing LC/MS system may be converted conveniently into a parallel LC/MS system with minimal modification. An equally important aspect of this high throughput ADME system was the development of a number of custom AppleScripts and Excel macros and templates to streamline and automate the pre- and postanalysis processing of massive amounts of experimental data. For example, for every plate of samples (88 compounds +8 controls) 
requiring microsomal stability analysis, more than 1000 samples are generated, requiring sophisticated data processing. Only with these data acquisition and postdata acquisition processing tools is it possible to make the system truly high throughput. The system can analyze 240 or more samples per hour. As the first application of the system, we developed a high throughput microsomal metabolic stability assay protocol. Using this protocol we are able to generate microsomal stability profiles of up to 176 test compounds per day (two microtiter plates of samples) at four time points $\left(t_{0}, t_{5}, t_{15}, t_{30}\right)$ and in triplicate (a total of 12 discrete samples required for analysis for each test compound).

\section{Experimental}

\section{Chemicals and Reagents}

Acetonitrile (ACN) was obtained from J. T. Baker (Phillipsburg, NJ). Dimethylsulfoxide (DMSO) was obtained from EM Science (Gibbstown, NJ). Formic acid, trichloroacetic acid (TCA), sodium phosphate, sodium hydroxide $(\mathrm{NaOH}), \mathrm{D}$-nicotinamide adenine dinucleotide phosphate reduced form (NADPH), atenolol, buspirone hydrochloride, imipramine hydrochloride, lidocaine hydrochloride, methoxyverapamil hydrochloride, DLpropranolol hydrochloride, $( \pm)$-sulpiride, and ( \pm )-verapamil hydrochloride were obtained from Sigma Chemical Co. (St. Louis, MO). In these studies, pooled human liver microsomes (HLM's) containing $20 \mathrm{mg} / \mathrm{mL}$ of total protein were used and obtained from Gentest Corp. (Woburn, MA). Sodium phosphate buffer was prepared as a $50 \mathrm{mM}$ stock solution in deionized water and adjusted with $1 \mathrm{M} \mathrm{NaOH}$ solution to $\mathrm{pH}$ 7.4. The stock reference solutions (atenolol, buspirone, imipramine, lidocaine, methoxyverapamil, propranolol, sulpiride, and verapamil) were prepared at $10 \mathrm{mM}$ concentration in DMSO, then diluted to $1 \mathrm{mM}$ with ACN prior to use. NADPH solution was prepared at 4 $\mathrm{mM}$ concentration in phosphate buffer prior to use. TCA solution was prepared at $0.3 \mathrm{M}$ in deionized water. The microsomes were thawed and mixed with phosphate buffer to give a $2 \mathrm{mg} / \mathrm{mL}$ total protein solution before use.

\section{High Throughput Metabolic Stability Procedure}

This procedure has been applied to the metabolic stability studies of compounds in liver microsomes from various species, including human, monkey, dog, rat, and mouse, although only human liver microsome experiments are described in this article. The following procedure was applied to 96-well microtiter plate microsomal incubations, schematically represented in Figure 1. Eight reference compounds (atenolol, buspirone, imipramine, lidocaine, methoxyverapamil, propranolol, sulpiride, and verapamil) were incubated with 88 test compounds in a 96-well plate. Liquid transfer steps

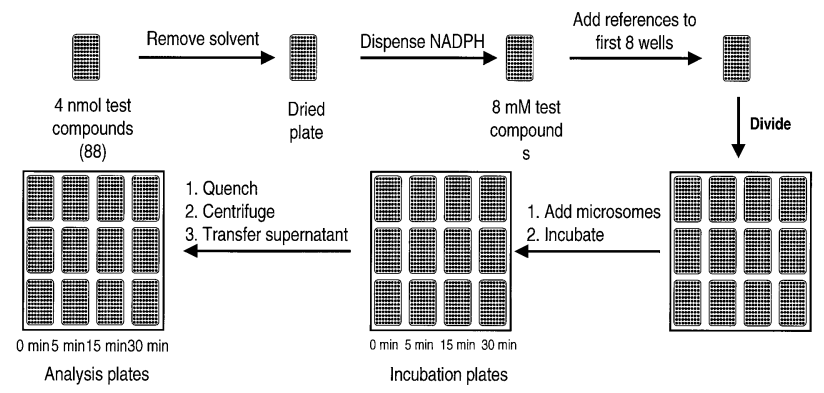

Figure 1. High throughput microsomal stability experiment procedure conducted in 96-well plate format. Each plate of test compounds yields twelve plates of analysis samples when the experiment is conducted in triplicate at four time points $(0,5,15$, $30 \mathrm{~min})$

were performed with a PP-550 MS 96-channel Personal Pipettor (Apricot Designs, Inc., Monrovia, CA) under computer control. Test compounds (4 nanomoles each) were dispensed, by aid of a Tecan Genesis Robotic Sample Processor (Tecan, Hombrechtikon, Switzerland), into 88 wells of a $1.2-\mathrm{mL}$ deep-well substrate plate. Compounds were arrayed in the microtiter plate in such a way as to leave the first column (8 wells) empty for reference compounds. The plate was then dried down to remove the solvent by aid of speed-vac lyophilization (Savant, Inc., Farmingdale, NY). NADPH solution $(500 \mu \mathrm{L}, 4 \mathrm{mM})$ was then added to each well of the plate to dissolve the test compounds. In some instances, it was necessary to agitate and heat the plates to aid in dissolution, as some compounds do not readily dissolve in the NADPH buffer solution. Solutions of eight reference compounds $(4 \mu \mathrm{L} 1 \mathrm{mM})$ were added into the first column of the microtiter plate. The solutions in the plate were then transferred into twelve 200 $\mu \mathrm{L}$ low volume incubation plates (30 $\mu \mathrm{L}$ each well). The incubation plates and the microsomes plate $(2 \mathrm{mg} / \mathrm{mL}$ total protein) were preheated at $37^{\circ} \mathrm{C}$ for $5 \mathrm{~min}$ in a VWR incubator (VWR Scientific Products, Cornelius, $\mathrm{OR})$. The $2 \mathrm{mg} / \mathrm{mL}$ microsomes solution was then added into all wells of the incubation plates $(30 \mu \mathrm{L} /$ well). The final incubation solutions contain $4 \mu \mathrm{M}$ test compound or reference compound, $2 \mathrm{mM}$ NADPH, 1 $\mathrm{mg} / \mathrm{mL}$ (total protein) microsomes, $50 \mathrm{mM}$ phosphate ( $\mathrm{pH}$ 7.4). The incubation plates were then incubated at $37^{\circ} \mathrm{C}$ for $0,5,15$, and $30 \mathrm{~min}$, respectively. At each time point, three incubation plates were removed from the incubator and quenched immediately.

Several different solvents were evaluated for quenching the microsomal stability incubations. Typically, acetonitrile is added in equal or excess volume to the microsomal incubate (i.e., NADPH buffer solution containing microsomes) to quench the reaction and precipitate proteinaceous material. However, it was found that a significant number of the test compounds eluted in the solvent front upon injection onto the short C18 analytical columns. It was found that an equal volume of aqueous $0.3 \mathrm{M}$ TCA was effective at quenching the enzyme activity and precipitating the proteins. 


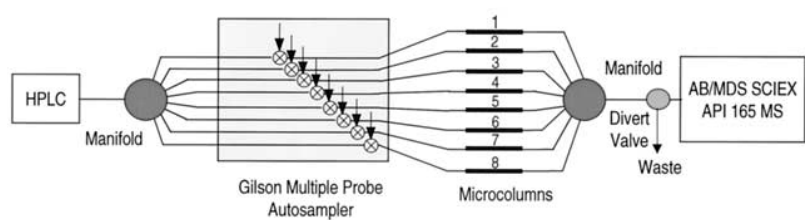

Figure 2. High throughput eight-channel parallel LC/MS system consisting of Shimadzu gradient HPLC pumps, a Gilson 215 multiple probe autosampler, an AB/MDS-SCIEX API165 Mass Spectrometer equipped with a TurboIonSpray ion source, eight Peeke Scientific microbore columns $(10 \mathrm{~mm} \times 1 \mathrm{~mm}$ i.d., $3 \mu \mathrm{m}$, HQ-C18), and a Valco switching valve. The volume of the sample loops is $20 \mu \mathrm{L}$ and full loop injections were used for all experiments. Total mobile phase flow rate is $2.0 \mathrm{~mL} / \mathrm{min}(0.25 \mathrm{~mL} / \mathrm{min}$ for each column). The flow is diverted to waste by a Valco switching valve in the early part of the gradient.

This solution also drastically reduced the number and amount of test compounds eluting in the void volume. A number of control samples were evaluated for chemical stability in the presence of this 50/50 NADPH buffer/TCA solution and none were found to have degraded. Therefore, $60 \mu \mathrm{L}$ of $0.3 \mathrm{M}$ TCA was added to each well to quench the reaction. The quenched plates were centrifuged for $15 \mathrm{~min}$ in a SpeedVac Plus centrifuge. The supernatants were then transferred to new $200 \mu \mathrm{L}$ low volume analytical plates for high throughput parallel LC/MS.

\section{High Throughput Parallel LC/MS}

The high throughput parallel LC/MS system shown in Figure 2 consists of two Shimadzu LC10ADvp Solvent Delivery pumps, an SCL-10Avp System Controller (Shimadzu Corp. Columbia, MD), a Gilson 215 multiple probe autosampler (8-channel) (Gilson, Inc., Madison, WI), a Valco switching valve (Valco Instruments Co. Inc., Houston, TX), and an Applied Biosystems/MDS-SCIEX API165 single quadrupole mass spectrometer equipped with a TurboIonSpray ion source (AB/MDS-SCIEX, Foster City, CA). The autosampler has eight needles spaced to fit eight wells of a standard 96-well microtiter plate. Eight samples are injected into the eight injection ports simultaneously and onto eight separate microbore columns. The volume of the sample loops is $20 \mu \mathrm{L}$ and full loop injections were used for all experiments. A total mobile phase flow rate of $2.0 \mathrm{~mL} / \mathrm{min}$ was used and split into eight equivalent streams using a Valco manifold before entering the multiple probe autosampler. Eight microbore columns $(10 \mathrm{~mm} \times 1 \mathrm{~mm}$ i.d., $3 \mu \mathrm{m}$, HQ-C18, Peeke Scientific, Redwood City, CA) were connected to eight injection valves of the autosampler (flow rate $0.25 \mathrm{~mL} / \mathrm{min}$ for each column). The outlets of the columns were recombined using a second Valco manifold. The flow was then passed through a Valco flow divert valve before entering the mass spectrometer. The in-line flow divert valve was used to ensure that undesirable materials eluted at the solvent front would be di- verted to waste to keep the ion source from becoming contaminated. When the valve was in the sampling position, the mobile phase was passed directly into TurbolonSpray ion source without splitting. The ion source was maintained at $350{ }^{\circ} \mathrm{C}$ to ensure sufficient desolvation of the liquid droplets in the ion source at the total $2 \mathrm{~mL} / \mathrm{min}$ flow rate. The mobile phase solvents used in this study were (A) $0.04 \%$ formic acid in water; and (B) $0.04 \%$ formic acid in acetonitrile. The following mobile phase gradient was applied: $1 \%$ B hold for $0.3 \mathrm{~min} ; 1-25 \% \mathrm{~B}$ in $0.05 \mathrm{~min}$; hold at $25 \%$ B for $0.1 \mathrm{~min} ; 25-70 \%$ B in $0.4 \mathrm{~min}$; hold at $70 \% \mathrm{~B}$ for $0.4 \mathrm{~min}$. The switching valve was set to divert flow to waste for the first $0.25 \mathrm{~min}$ of the gradient. The columns were reequilibrated at starting conditions while the next set of samples was injected. Two computers were used to acquire microsomal stability data. The AB/MDS-SCIEX API165 mass spectrometer was controlled by a G3 Power Macintosh and associated MassChrom 1.2 software. The autosampler was controlled by a PC with UniPoint software (Gilson, Inc., Madison, WI). The two computers communicated with each other through contact closures for synchronized operation. The mass spectrometer was configured to acquire data in selected ion monitoring (SIM) mode, allowing eight ions to be monitored during each scan (dwell time 50

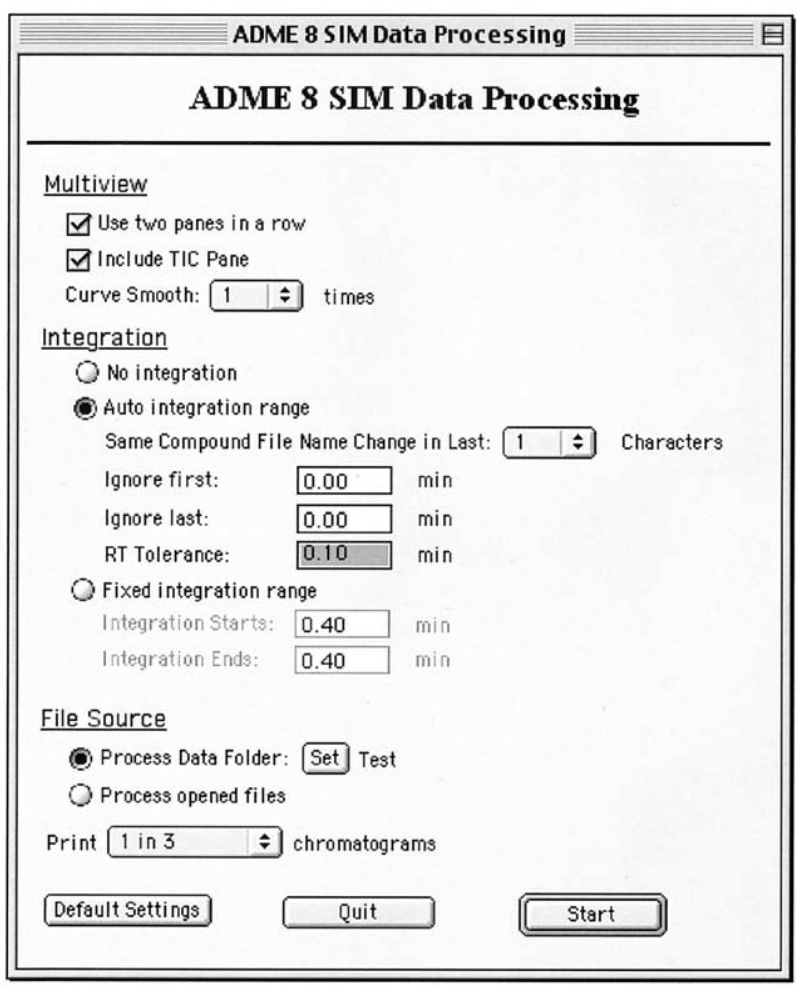

Figure 3. ADME 8 SIM Data Processing script batch processes LC/MS data using an intelligent algorithm to ensure the peaks of interest are integrated consistently and correctly. It extracts and integrates all SIM chromatograms in each file and writes the integration results to an output file that is then imported into Excel templates for final report generation. 


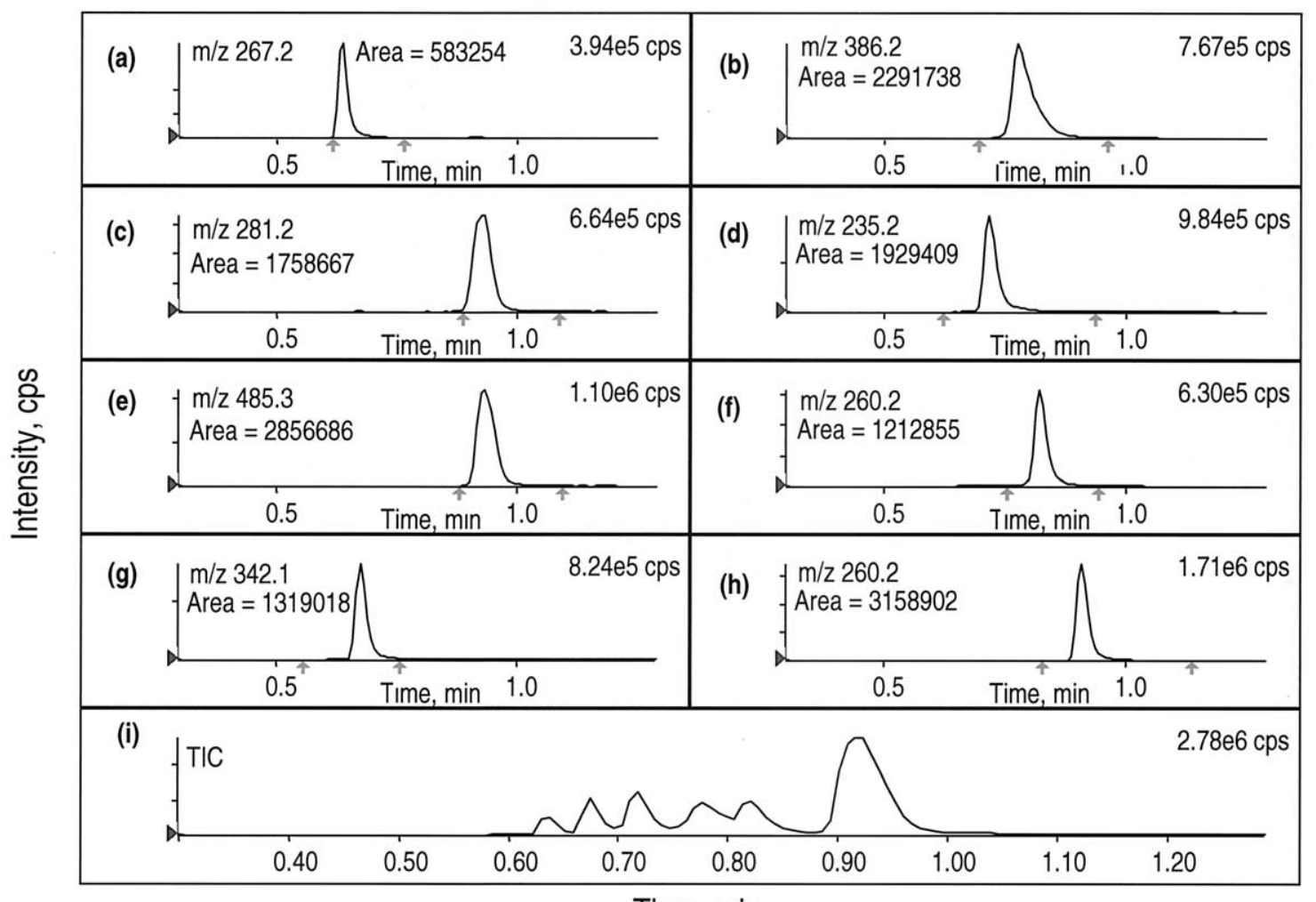

Time, $\min$

Figure 4. An example of the selected ion monitoring chromatograms from the eight-column parallel analysis. The trace of each compound was extracted from the total ion chromatogram by the ADME 8 SIM Data Processing script. (a) Verapamil, (b) propranolol, (c) imipramine, (d) lidocaine, (e) sulpiride, (f) atenolol, (g) buspirone, (h) methoxyverapamil, and (i) total ion chromatogram of all eight compounds.

ms for each ion). The SIM values were defined in the job list of the MassChrom 1.2 Sample Control data acquisition sheet. All data were acquired in the positive ionization mode. The instrument resolution was set to 1500 (10\% valley definition) based on the infusion of a (poly)propylene glycol calibrant solution.

\section{Scripts and Macros are Critical to Successful High Throughput ADME Analyses}

Several custom AppleScripts, Excel macros, and Excel templates were designed and developed to facilitate both pre- and postanalysis processing in order to take full advantage of this system for high throughput

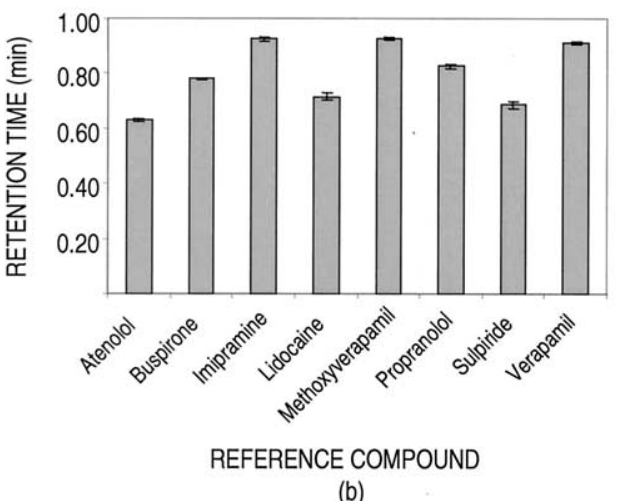

(b)

Figure 5. Assessment of intra-column performance in parallel mode of operation. Each compound $(0.5 \mu \mathrm{M})$ is injected onto a unique column with 500 repeat injections over a period of $17 \mathrm{~h}$. The coefficient of variations (CV) of retention times and mass spectrometric peak areas for all of the eight columns are below $2 \%$ (average $0.9 \%$ ) and 10\% (average 5.7\%), respectively. (a) Peak areas and (b) retention times. 


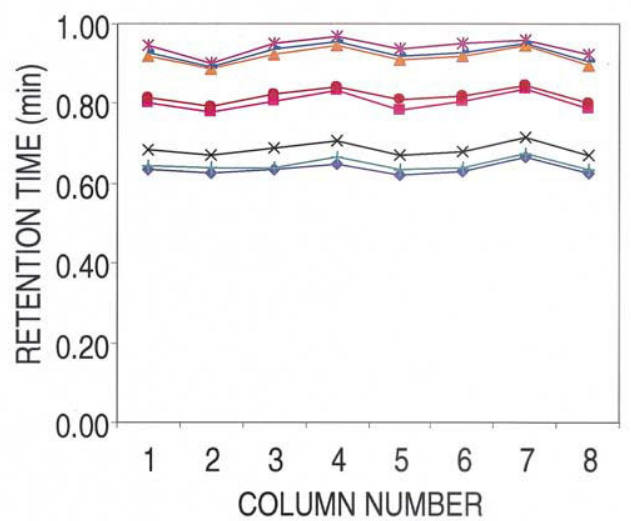

(a)
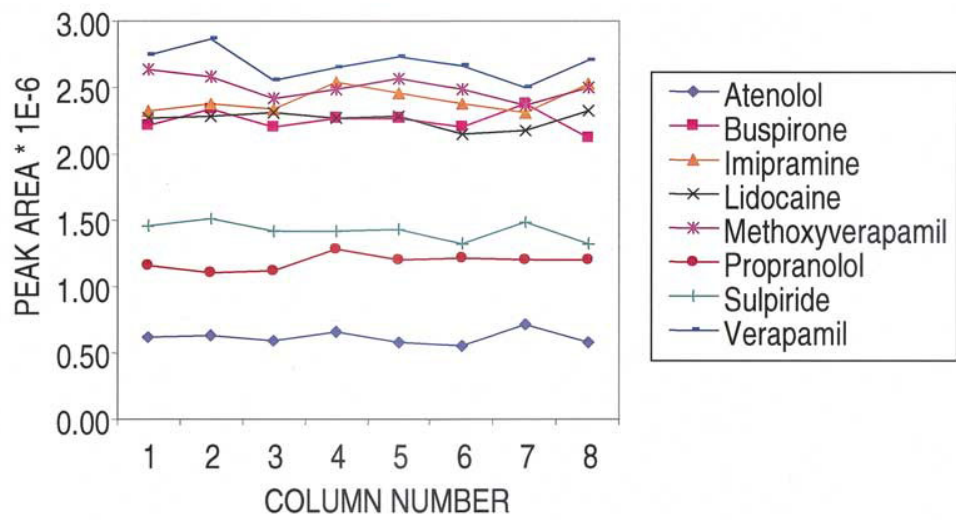

(b)

Figure 6. Assessment of inter-column variability of retention times and peak areas using eight parallel columns and a binary pumping system with a precolumn flow splitter. A solution containing $0.5 \mu \mathrm{M}$ of the eight reference compounds was injected sequentially onto each of the eight columns while the remaining seven columns received blank injections. The data of five replicate injections show that the coefficient of variations $(\mathrm{CV})$ of retention times and peak areas among the eight columns are below $2 \%$ (average $0.5 \%$ ) and 10\% (average 3.6\%), respectively. (a) Retention times and (b) peak areas.
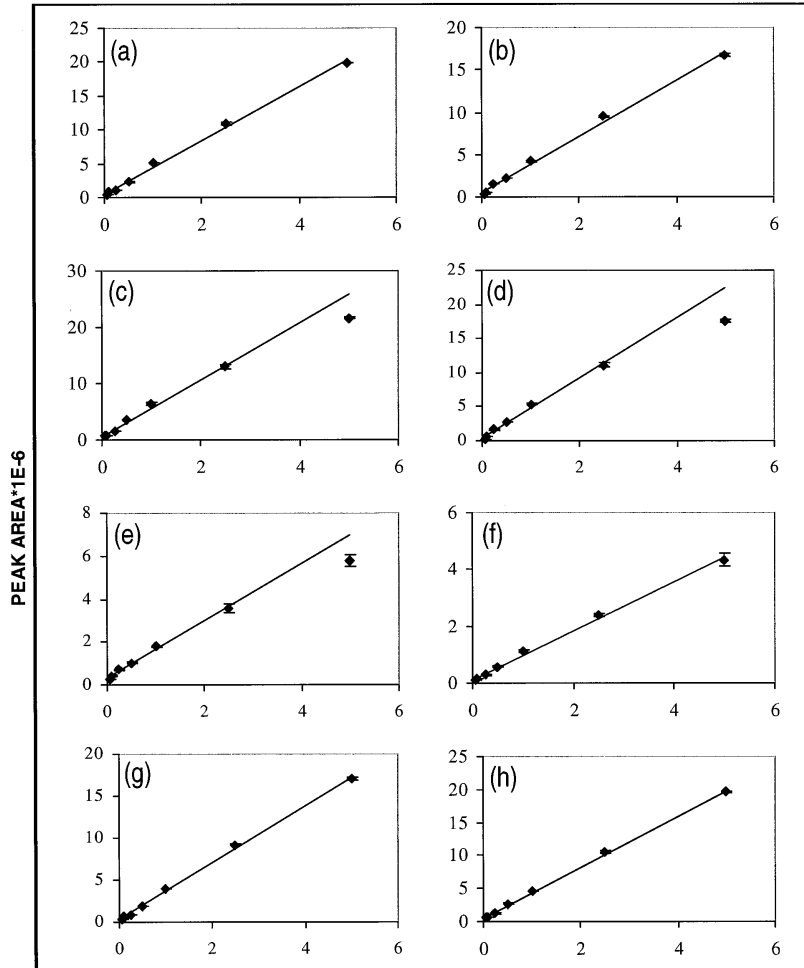

CONCENTRATION (UM)

Figure 7. Linear correlation of the peak areas with sample concentrations in the range from 0.05 to $5 \mu \mathrm{M}$ (injection volume: $20 \mu \mathrm{L}$ ). Each concentration was injected in triplicate. The linear correlation curves were excellent between 0.05 and $2.5 \mu \mathrm{M}$ for all eight references and could be extended to $5 \mu \mathrm{M}$ or above for most of the compounds. (a) Atenolol, (b) buspirone, (c) imipramine, (d) lidocaine, (e) methoxyverapamil, (f) propranolol, (g) sulpiride, and (h) verapamil. microsomal stability assays. The first of these was a FormatPlate Excel macro. The macro first compares SIM values of each of the test compounds in the set. Next, it arranges the compounds in appropriate wells such that the molecular weights are no closer than three Da from one another (obviating the need for indexed MUX analysis). For almost all compounds evaluated, this 3 Da window has been satisfactory. In those instances where a compound was found to contain more than one chlorine and/or bromine atom, a larger mass Da window between samples was used. A FormatSampleControl macro generates a job list for Sample Control to execute the LC/MS analysis. For data processing, an ADME 8 SIM Data Processing script (Figure 3) was developed. The script launches MultiView, opens each data file, extracts and integrates all SIM chromatograms in each file and writes the integration results to an output file that is imported into Excel templates for final report generation. An algorithm in the ADME 8 SIM Data Processing script was also developed to ensure the peaks of interest are integrated consistently and correctly, as will be discussed shortly. A StabilityReport macro was developed to import the validated integration result file into an Excel Report Template and automatically generate a report with plots, final results, and data quality evaluations. Features of these scripts and macros are discussed below.

\section{Results and Discussion}

\section{Intra- and Inter-Column Retention Times and Peak Area Variation Among the Eight Columns}

Much discussion has ensued as to whether an HPLC system can be conveniently converted from serial mode 


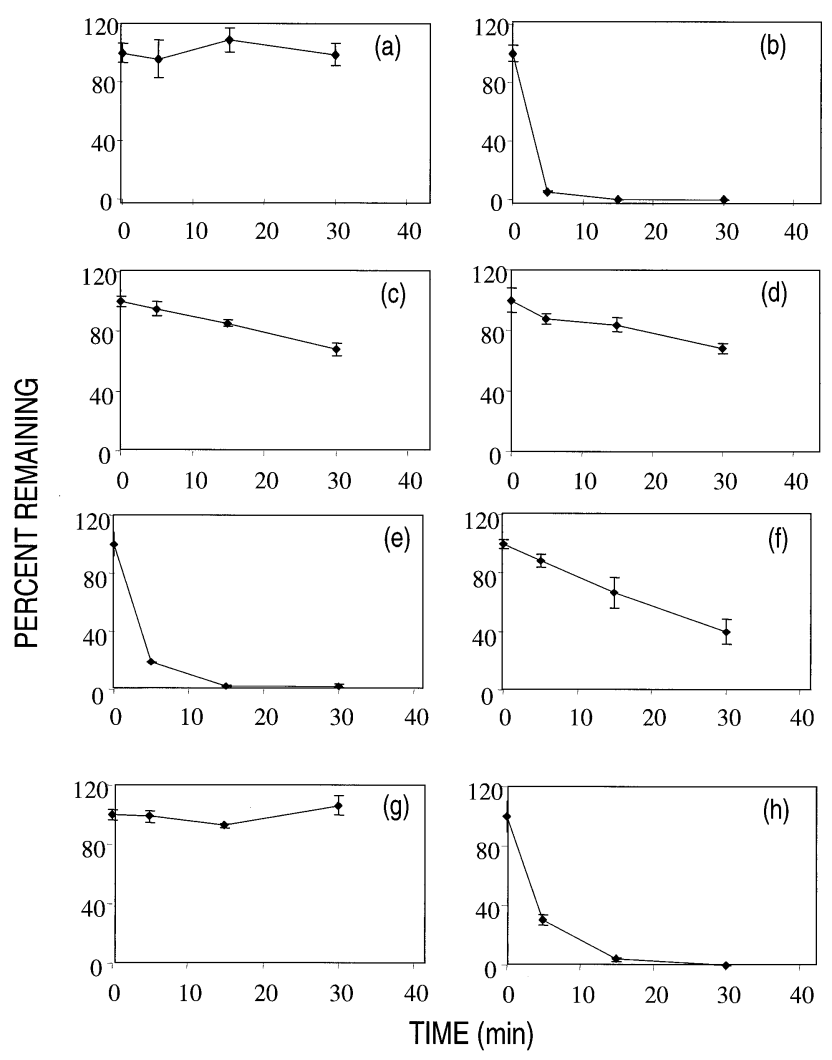

Figure 8. Microsomal stability profiles of eight reference compounds. (a) Atenolol, (b) buspirone, (c) imipramine, (d) lidocaine, (e) methoxyverapamil, (f) propranolol, (g) sulpiride, and (h) verapamil. Buspirone, methoxyverapamil, and verapamil showed fast turnover; imipramine, lidocaine, and propranolol intermediate; and atenolol and sulpride slow.

to parallel mode without sophisticated pumping and flow control. In our laboratory, successful parallel analytical LC/MS has been achieved using a simple valco manifold to split the flow from a binary HPLC system evenly between eight columns. Approximately 100,000 samples have been analyzed in this parallel configuration for early ADME assessment successfully.

A series of experiments were conducted to test the intra-column variation when operated in the parallel mode. System performance was evaluated using the eight reference compounds: atenolol $(\mathrm{m} / \mathrm{z} 267.2)$, buspirone $(\mathrm{m} / \mathrm{z} 386.2)$, imipramine $(\mathrm{m} / \mathrm{z} 281.2)$, lidocaine $(\mathrm{m} / \mathrm{z}$ 235.2), methoxyverapamil ( $\mathrm{m} / \mathrm{z} 485.3)$, propranolol $(\mathrm{m} / \mathrm{z}$ 260.2), sulpiride $(m / z 342.1)$, and verapamil $(m / z 455.3)$. Eight solutions containing $0.5 \mu \mathrm{M}$ of each individual reference compound were prepared and injected onto the eight columns simultaneously. The experiment was performed over a period of $17 \mathrm{~h}$ with a total of 500 repeat injections. An example of the eight chromatograms from the eight column parallel analysis is shown in Figure 4. The data show that the coefficient of variations $(\mathrm{CV})$ of retention times and mass spectrometric peak areas for all of the eight columns are below $2 \%$ (average $0.9 \%$ ) and $10 \%$ (average $5.7 \%$ ), respectively (Figure 5).
To assess inter-column variation, a solution containing $0.5 \mu \mathrm{M}$ of the eight reference compounds was prepared and injected sequentially onto each of the eight columns while the remaining seven columns received blank injections. A total of five replicate injections was made. The data for the eight reference compounds show that the coefficient of variations $(\mathrm{CV})$ of retention times and peak areas among the eight columns are below $2 \%$ (average $0.5 \%$ ) and $10 \%$ (average $3.6 \%$ ), respectively (Figure 6). This data shows that the valco flow splitter is effective at providing equal flows to each of the eight columns.

\section{Linear Correlation of the Peak Areas with Sample Concentrations}

Standard solutions of the eight reference compounds were prepared at eight concentrations in the range from 0.05 to $5 \mu \mathrm{M}$ (injection volume: $20 \mu \mathrm{L}$ ). Each concentration was injected in triplicate. The linear correlation curves were excellent between 0.05 and $2.5 \mu \mathrm{M}$ for all eight references and could be extended to $5 \mu \mathrm{M}$ or above for most of the compounds (Figure 7). It was assumed that similar linear correlations would be observed for the test compounds and that there was therefore no need to construct individual calibration curves for each of the test compounds. Instead, the relative percent remaining of the parent compounds was calculated by directly comparing samples at each time point $\left(t_{5}, t_{15}\right.$, and $\left.t_{30}\right)$ to its initial time point $\left(t_{0}\right)$.

\section{High Throughput Microsomal Stability Profiling of Compound Libraries}

High throughput microsomal stability experiments of project compounds are performed without any preanalysis or prequalification in order to ensure rapid turnaround to the project teams. For each plate of test compounds, more than one thousand samples are generated for LC/MS analysis. In the absence of automated data processing tools, the postanalysis data reduction and validation processes would be exceedingly tedious. To address this data management problem, a StabilityReport macro was developed to automate these tasks. The macro imports the integration result file, deconvolutes the eight-channel results, and generates stability plots for each compound as well as a summary report for the whole plate. The macro then further analyzes and validates the results of each compound, generating a flag for any compound that has incorrect stability trend, low MS signal, or broad chromatographic peak. With this intelligent validation tool, the postanalysis data processing time is automatically reduced from about one day per plate (manually) to literally minutes per plate. A sample plot of the eight reference standards is shown in Figure 8. Buspirone, methoxyverapamil, and verapamil showed fast turnover; imipramine, lido- 


\section{$108325(031401)$}

\begin{tabular}{|c|c|c|c|c|}
\hline Protocol: & ALMA_1.0 & & & \\
\hline Compounds & SIMI & 残(5 min) & \%(15 min) & \%(30 min) \\
\hline Atenolol & 267.2 & 100.0 & 100.0 & 100.0 \\
\hline Buspirone & 386.2 & 5.4 & 0.0 & 0.1 \\
\hline Imipramine & 281.2 & 95.2 & 85.6 & 68.5 \\
\hline Lidocaine & 235.2 & 87.6 & 83.7 & 68.3 \\
\hline Methoxyverapamil & 485.3 & 18.6 & 2.5 & 2.5 \\
\hline Propranolol & 260.2 & 88.5 & 66.8 & 40.1 \\
\hline Sulpiride & 342.1 & 98.7 & 92.7 & 106.3 \\
\hline Verapamil & 455.3 & 30.4 & 4.2 & 0.0 \\
\hline 613678 & 346.0 & 95.1 & 107.5 & 112.3 \\
\hline 613592 & 331.0 & 108.1 & 115.1 & 104.2 \\
\hline 613676 & 333.0 & 94.1 & 97.0 & 103.1 \\
\hline 613793 & 466.0 & 112.8 & 106.7 & 101.6 \\
\hline 613603 & 391.0 & 100.0 & 100.0 & 100.0 \\
\hline 613672 & 365.0 & 100.0 & 100.0 & 100.0 \\
\hline 613679 & 333.012 & 92.9 & 86.5 & 91 \\
\hline 613626 & 377.0 & 94.8 & 93.2 & 71.5 \\
\hline 613673 & 317.0 & 93.6 & 84.5 & 68.6 \\
\hline 613807 & 331.0 & 73.1 & 47.3 & 53.8 \\
\hline 613645 & 380.0 & 82.2 & 70.5 & 50.9 \\
\hline 613602 & 369.0 & 86.6 & 67.8 & 44.4 \\
\hline 613599 & 369.0 & 80.1 & 59.8 & 40.9 \\
\hline 613671 & 319.0 & 80.2 & 55.6 & 36. \\
\hline 613605 & 415.0 & 62.4 & 37.3 & 34.9 \\
\hline 613808 & 345.0 & 81.4 & 64.2 & 31. \\
\hline 613608 & 375.0 & 89.7 & 55.4 & 29.9 \\
\hline 613670 & 289.0 & 76.3 & 47.9 & 28.5 \\
\hline 613638 & 353.0 & 55.3 & 21.5 & 25.5 \\
\hline 613641 & 313.0 & 65.1 & 45.2 & 20.4 \\
\hline 613613 & 429.0 & 43.0 & 10.7 & 20.2 \\
\hline 613609 & 415.0 & 16.3 & 2.8 & 16.1 \\
\hline
\end{tabular}

Figure 9. A partial microsomal stability summary report of a plate of eight reference compounds and 88 test compounds received from a drug discovery project and tested without any preanalysis or prequalification. The percent remaining values of the parent compounds were colored for easy visualization: Green, $>80 \%$; yellow, $80-40 \%$; red, $<40 \%$; gray, flagged by the intelligent validation macro.

caine, and propranolol intermediate; and atenolol and sulpiride slow.

As can be seen from the data in Figure 8, the amount of parent remaining at the end of a microsomal incubation may be very high or very low, depending on whether or not the compound is a substrate for the cytochrome P450 enzymes. For example, buspirone and verapamil (Figure $8 \mathrm{~b}$ and $\mathrm{h}$ respectively) exhibit less than $5 \%$ parent remaining after a $30 \mathrm{~min}$ incubation. At an initial substrate concentration of $2 \mu \mathrm{M}, 5 \%$ remaining corresponds to a concentration of $0.1 \mu \mathrm{M}$ (or 100 femtomole $/ \mu \mathrm{L}$ concentration). At this concentration, the chromatographic peak of interest very often may not be integrated properly. The low intensity peaks can be affected by baseline noise and result in improper integration. To address this problem, an algorithm in the ADME 8 SIM Data Processing script was developed to ensure the peaks of interest are integrated consistently and correctly. Files were named systematically so that the script recognizes all files corresponding to the same set of compounds. The script uses MassChrom's integration tool to obtain the retention times at the start of the peak, the apex of the peak, and at the end of the peak, specifically for the $t_{0}$ time-point for each set of compounds. Since the $t_{0}$ time-point contains the most amount of parent and gives the strongest ion intensity, the script applies these start, end, and peak max values to the remaining time-points automatically. If the peak apex moves in this narrowly defined time range because of slight chromatographic condition changes, the 

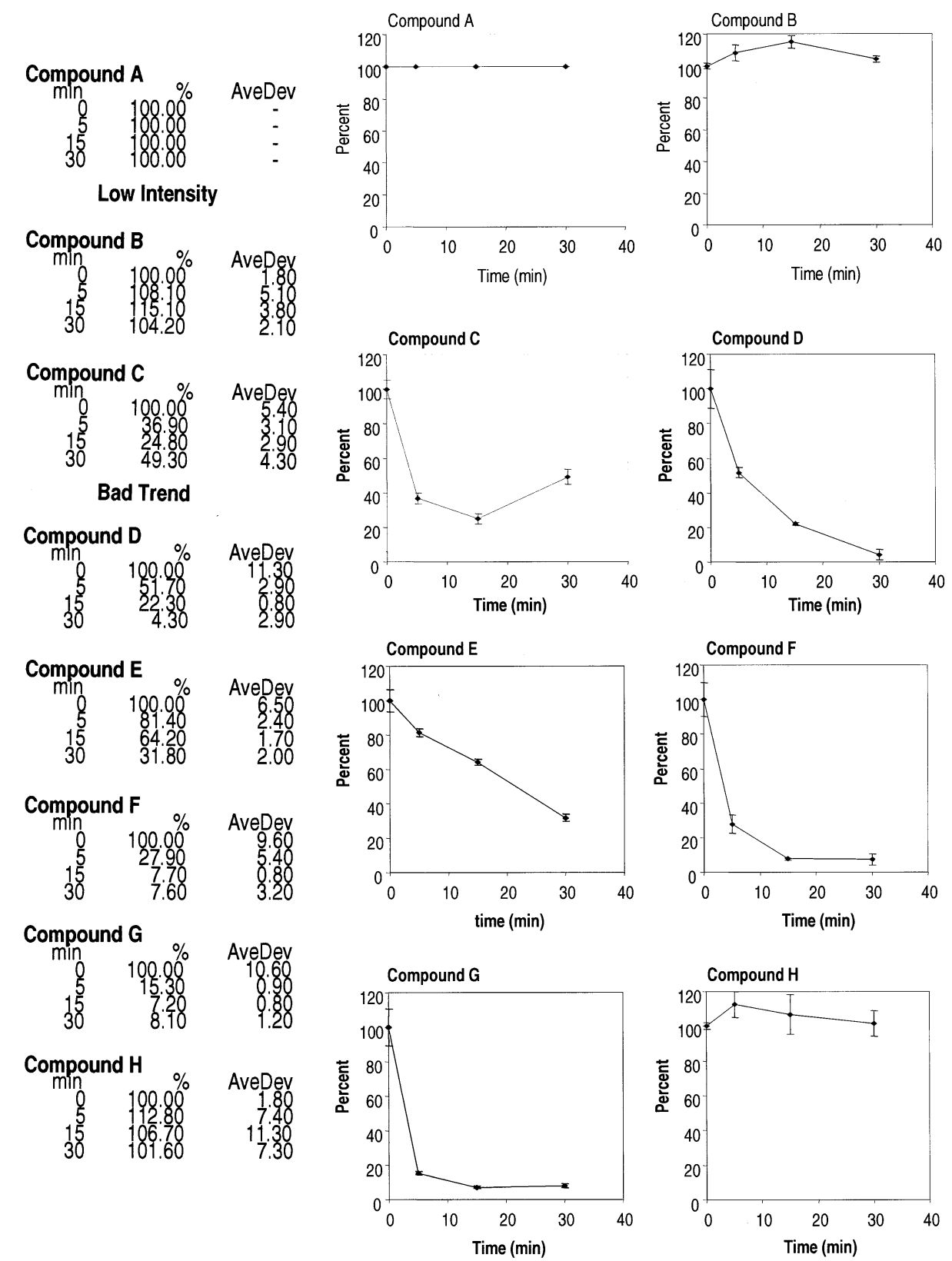

Figure 10. A microsomal stability report of eight test compounds received from a drug discovery project and tested without any preanalysis or prequalification. Compounds D, F and G showed fast turnover; Compound $\mathrm{E}$ intermediate; and Compounds B and $\mathrm{H}$ slow. As identified by the intelligent validation macro and noted next to the plots, Compound A did not yield a profile because of low ion intensity, and Compound C showed a poor stability trend.

script will automatically apply an offset to the integration window so that the peak is integrated properly. Since the integration is performed in a fixed window determined by the script for each compound, Multiview's integration tool can not be used. The script uses its own integration routine that integrates the area point by point after subtracting the background. This integration routine was validated against Multiview's integration tool using the same integration window and gave similar results.

A microsomal stability partial summary report of a plate of 8 reference compounds and 88 test compounds received from a drug discovery project and tested without any preanalysis or prequalification is shown in Figure 9. The percent remaining values of the parent compounds were color-coded for easy visualization: Green, $>80 \%$; yellow, $80-40 \%$; red, $<40 \%$; gray, flagged by the intelligent validation macro. Figure 10 shows a report page of the microsomal stability profiles of eight test compounds. Compounds D, F, and G showed fast turnover, Compound $\mathrm{E}$ intermediate, and Compounds $\mathrm{B}$ and $\mathrm{H}$ slow. As identified by the intelli- 
gent validation macro and noted next to the plots, Compound A did not yield a profile due to low ion intensity and Compound $\mathrm{C}$ showed a poor stability trend. The project chemist receives both the summary report which helps "bin" the compounds into distinct classes of microsomal stability and the time-course stability plots for all compounds submitted for HT microsomal stability analysis. This information helps the chemists prioritize compounds for further consideration as potential drug candidates.

\section{Conclusions}

An eight-channel parallel LC/MS system was presented for high throughput analysis of compound libraries for microsomal stability, permitting up to 240 samples to be analyzed per hour. The high throughput system was shown to perform exceedingly well in terms of reproducibility and robustness. Only very small column to column variations of retention times and peak areas were observed. The linear range was satisfactory for the microsomal stability experiments described in this article (maximum concentration $2 \mu \mathrm{M}$ ). The system is easily modified for parallel operation on existing LC/MS instruments requiring only minimal hardware modifications and has been applied successfully to the microsomal stability profiling of over 10,000 project compounds.

Acetonitrile is the most commonly used solvent for quenching and precipitating the microsomal incubations. However, we found that a number of compounds dissolved in 50/50 NADPH/acetonitrile buffer eluted in the solvent front upon injection onto the $10 \mathrm{~mm} \times 1$ $\mathrm{mm}$ i.d. C18 columns. TCA, on the other hand, was found effective at quenching the microsomal incubations while enabling compounds to be retained on column. A potential problem with using an acid as a quench solution is that the compounds may undergo chemical degradation. None of our standards was found to degrade in the presence of this TCA solution. One way to confirm that none of the test compounds is degraded when exposed to this TCA quenching solution is to incubate the test compounds in the absence of $\mathrm{NADPH}$, a cofactor required for enzymatic activity). The relative response of the test compound in the presence/absence of NADPH would allow one to readily decouple chemical stability from microsomal stability.

It is assumed that positive electrospray ionization detection and our so called universal gradient are satisfactory for the vast majority of compounds contained within the library. In reality, we observe on average a $65-70 \%$ success rate. The majority of failures are attributable to weak ion intensities, poor compound solubility, and/or experimental pipetting or injection errors. Of these three, solubility appears to have the greatest impact on the success rate to date. Additional limitations of this system are mainly (1), signal reduction due to recombination of eight streams, and (2), mass separation requirement in the same set of injections. The former is not a problem for in vitro ADME assays of most pharmaceutical compounds but does sometimes cause quantification difficulty for poorly ionizable compounds. The latter has to be handled in the sample submission step to ensure test compounds are plated properly. With further development, MUX electrospray interfaces that introduce multiple LC flows directly into ion sources and employ indexing to differentiate individual channels may eliminate some of the above limitations and further improve the success rate and the workflow efficiency.

\section{Acknowledgments}

The authors are grateful to Mr. Tao Wang, DuPont Pharmaceutical Research Laboratories, for providing useful suggestions to enhance the robustness of the technique for HT ADME of compound libraries.

\section{References}

1. Watt, A. P.; Morrison, D.; Evans, D. C. Approaches to Higher Throughput Pharmacokinetics (HTPK) in Drug Discovery. Drug Disc. Today 2000, 5, 17-24.

2. White, R. E. High Throughput Screening in Drug Metabolism and Pharmacokinetic Support of Drug Discovery. Annu. Rev. Pharmacol. Toxicol. 2000, 40, 133-157.

3. Kennedy, T. Managing the Drug Discovery/Development Interface. Drug Disc. Today 1997, 2, 436-444.

4. Sinko, P. J. Drug Selection in Early Drug Development: Screening for Acceptable Pharmacokinetic Properties Using Combined in Vitro and Computational Approaches. Curr. Opin. Drug Disc. Dev. 1999, 2, 42-48.

5. de Waterbeemd, H.; Smith, D. A.; Beaumont, K.; Walker, D. K. Property-Based Design: Optimization of Drug Absorption and Pharmacokinetics. J. Med. Chem. 2001, 44, 1313-1333.

6. Linget, J. M.; Vignaud, P. D. Automation of Metabolic Stability Studies in Microsomes, Cytosol and Plasma Using a 215 Gilson Liquid Handler. J. Pharm. Biomed. Anal. 1999, 19, 893-901.

7. Korfmacher, W. A.; Palmer, C. A.; Nardo, C.; Dunn-Meynell, K.; Grotz, D.; Cox, K.; Lin, C.-C.; Elicone, C.; Liu, C.; Duchoslav, E. Development of an Automated Mass Spectrometry System for the Quantitative Analysis of Liver Microsomal Incubation Samples: A Tool for Rapid Screening of New Compounds for Metabolic Stability. Rapid Commun. Mass Spectrom. 1999, 13, 901-907.

8. Ayrton, J.; Dear, G. J.; Leavens, W. J.; Mallett, D. N.; Plumb, R. S. Optimization and Routine Use of Generic Ultra-High Flow-Rate Liquid Chromatography with Mass Spectrometric Detection for the Direct Online Analysis of Pharmaceuticals in Plasma. J. Chromatogr. A 1998, 828, 199-207.

9. Scott, R. J.; Palmer, J.; Lewis, I. A. S.; Pleasance, S. Determination of a "GW Cocktail" of Cytochrome P450 Probe Substrates and Their Metabolites in Plasma and Urine Using Automated Solid Phase Extraction and Fast Gradient Liquid Chromatography Tandem Mass Spectrometry. Rapid Commun. Mass Spectrom. 1999, 13, 2305-2319.

10. Zweigebaum, J.; Heining, K.; Steinborner, S.; Wachs, T.; Henion, J. High Throughput Bioanalytical LC/MS/MS Determination of Benzodiazepines in Human Urine: 1000 Samples per 12 Hours. Anal. Chem. 1999, 71, 2294-2300.

11. Zweigebaum, J.; Henion, J. Bioanalytical High Throughput Selected Reaction Monitoring-LC/MS Determination of Se- 
lected Estrogen Receptor Modulators in Human Plasma: 2000 Samples/Day. Anal. Chem. 2000, 72, 2446-2454.

12. Stevenson, C. L.; Augustijns, P. F.; Hendren, R. W. Use of Caco-2 cells and LC/MS/MS to Screen a Peptide Combinatorial Library for Permeable Structures. Intl. J. Pharm. 1999, 177(1), 103-15.

13. Shaffer, J. E.; Adkison, K. K.; Halm, K.; Hedeen, K.; Berman, J. Use of N-in-One Dosing to Create an in vivo Pharmacokinetics Database for Use in Developing Structure-Pharmacokinetic Relationships. J. Pharm. Sci. 1999, 88, 313-318.

14. Cox, K.; Dunn-Meynell, K.; Korfmacher, W.; Broske, L.; Nomeir, A. A.; Lin, C. C.; Cayen, M. N.; Barr, W. H. Novel in Vivo Procedure for Rapid Pharmacokinetic Screening of Discovery Compounds in Rats. Drug Disc. Today 1999, 4, 232-237.

15. van Pelt, C. K.; Corso, T. N.; Schultz, G. A.; Lowes, S.; Henion, J. A Four-Column Parallel Chromatography System for Isocratic or Gradient LC/MS Analyses. Anal. Chem. 2001, 73, 582-588.

16. Rudewicz, P. J.; Yang, L. Novel Approaches to High Throughput Quantitative LC-MS/MS in a Regulated Environment. Am. Pharm. Rev. 2001, 4, 64-70.

17. $\mathrm{Wu}, \mathrm{J}$. T. The Development of a Staggered Parallel Separation Liquid Chromatography/Tandem Mass Spectrometry System with On-Line Extraction for High Throughout Screening of Drug Candidates in Biological Fluids. Rapid Commun. Mass Spectrom. 2001, 15, 73-81.

18. Janiszewski, J. S.; Rogers, K. J.; Whalen, K. M.; Cole, M. J.; Liston,, T. E.; Duchoslav, E.; Houda, H. G. A High Capacity LC/MS System for the Bioanalysis of Samples Generated from Plate-Based Metabolic Screening. Anal. Chem. 2001, 73, 14951501.

19. Xu, R. Nemes, C., Liu, C. Z. C., Kassel, D. B. A High Throughput Parallel LC/MS Method for Assessing Metabolic Stability and Permeability of Compound Libraries. Proceedings of the 47th ASMS Conference on Mass Spectrometry and Allied Topics; Dallas, TX, 1999.

20. Korfmacher, W. A.; Veals, J.; Dunn-Meynell, K.; Zhang, X.; Tucker, G.; Cox, K. A.; Lin, C. C. Demonstration of the Capabilities of a Parallel High Performance Liquid Chromatography Tandem Mass Spectrometry System for Use in the Analysis of Drug Discovery Plasma Samples. Rapid Commun. Mass Spectrom. 1999, 13, 1991-1998.
21. Hiller, D. L., Cole, R. O. The Use of Indexed Multi-Probe Electrospray Technology for Quantitation in Support of Drug Discovery ADME Screens. Proceedings of the 48th ASMS Conference on Mass Spectrometry and Allied Topics; Long Beach, CA, 2000.

22. Chesson, S. M., Collis, J. P., Dage, J. L. Application of the Eight Channel Multiplexed Electrospray to High Throughput in Vitro ADME Screens. Proceedings of the 48th ASMS Conference on Mass Spectrometry and Allied Topics; Long Beach, CA, 2000.

23. Hiller, D. L., Cole, R. O., Little, D., Hooper, A. High Throughput Quantitation Using Indexed Multi-Probe Electrospray Technology in Support of Drug Discovery. Proceedings of the 48th ASMS Conference on Mass Spectrometry and Allied Topics; Long Beach, CA, 2000.

24. Hiller, D. L.; Brockman, A. H.; Goulet, L.; Ahmed, A.; Cole, R. O.; Covey, T. Application of a Non-Indexed Dual Sprayer Pneumatically Assisted Electrospray Source to the High Throughput Quantitation of Target Compounds in Biological Fluids. Rapid Commun. Mass Spectrom. 2000, 14, 2034-2038.

25. Bayliss, M. K.; Little, D.; Mallett, D. N.; Plumb, R. S. Parallel Ultra-High Flow Rate Liquid Chromatography with Mass Spectrometric Detection Using a Multiplex Electrospray Source for Direct, Sensitive Determination of Pharmaceuticals in Plasma at Extremely High Throughput. Rapid Commun. Mass Spectrom. 2000, 14, 2039-2045.

26. Yang, L.; Mann, T. D.; Little, D.; Wu, N.; Clement, R. P.; Rudewicz, P. J. Evaluation of a Four-Channel Multiplexed Electrospray Triple Quadrupole Mass Spectrometer for the Simultaneous Validation of LC/MS/MS Methods in Four Different Preclinical Matrixes. Anal. Chem. 2001, 73, 1740-1747.

27. Wang, T.; Zeng, L.; Cohen, J.; Kassel, D. B. A Multiple Electrospray Interface for Parallel Mass Spectrometric Analyses of Compound Libraries. Comb. Chem. High Throughput Screen 1999, 2, 327-334.

28. Zeng, L.; Kassel, D. B. Developments of a Fully Automated Parallel HPLC/Mass Spectrometry System for the Analytical Characterization and Preparative Purification of Combinatorial Libraries. Anal. Chem. 1998, 70, 4380-4388.

29. Jenkins, K. M., Rourick, R. A., Xu, R., Kassel, D. B. High Throughput Cytochrome P450 Inhibition Assays Using Parallel LC/MS Methodologies. Proceedings of the 48th ASMS Conference on Mass Spectrometry and Allied Topics; Long Beach, CA, 2000. 